\title{
Early pregnancy mood before and during COVID-19 community restrictions among women of low socioeconomic status in New York City: a preliminary study
}

\author{
Michael E. Silverman ${ }^{1}$ (D) Cathryn Medeiros ${ }^{2} \cdot$ Laudy Burgos $^{3}$ \\ Received: 27 May 2020 / Accepted: 18 August 2020 / Published online: 25 August 2020 \\ (C) Springer-Verlag GmbH Austria, part of Springer Nature 2020
}

\begin{abstract}
To explore the mental health consequences of COVID-19-related social restrictions on pregnant women living in low socioeconomic status. Prenatal women appearing at the Mount Sinai Hospital Ambulatory Practice were screened for mood symptomatology from February 2, 2020, through June 12, 2020. An improvement in prenatal mood was observed following social restrictions compared to before the pandemic. The impact of COVID-19 remains largely unknown and may be useful towards understanding the needs of pregnant women living in poverty.
\end{abstract}

Keywords COVID19 $\cdot$ Prenatal $\cdot$ Mood $\cdot$ Stress $\cdot$ Minority $\cdot$ Socioeconomic status

\section{Introduction}

Addressing the mental health needs of pregnant women during the Coronavirus disease 2019 (COVID-19) pandemic is an increasing concern among obstetricians and mental health clinicians. According to a recent Kaiser Family Foundation poll (2020), the majority of adults report their lives have been disrupted by the virus $(84 \%)$, and nearly half $(45 \%)$ report their mental health has been negatively impacted. While minority women living in low socioeconomic status (SES) are among the most vulnerable for mood disruption during pregnancy (Shakeel et al. 2015), how the COVID-19 pandemic, and accompanying social restrictions, may impact this population remains unknown.

This article is part of the Topical Collection on Women's mental health during the Covid-19 pandemic

Edited by: Anita Riecher-Rössler

Michael E. Silverman

Michael.silverman@mssm.edu

1 Department of Psychiatry, Icahn School of Medicine at Mount Sinai, The Mount Sinai Hospital, New York, NY, USA

2 Department of Social Work Services, The Mount Sinai Hospital, New York, NY, USA

3 Department of Social Work Services, Icahn School of Medicine at Mount Sinai, The Mount Sinai Hospital, New York, NY, USA
This study, therefore, aimed to explore how social restrictions associated with the COVID-19 pandemic may be impacting prenatal women living in low SES. The results of this exploration will help better understand the needs of women in poverty, both during the pandemic and thereafter.

\section{Methods}

In 2011, the Mount Sinai Hospital Obstetrics \& Gynecology Ambulatory Practice initiated the first large-scale universal symptomatology screening for perinatal mood utilizing clinical decision support tied into electronic medical records (Loudon et al. 2016). In February of 2020, to further improve clinical services, the assessment of mood at a woman's first prenatal visit was added.

\section{Sample characteristics}

The study cohort consisted of the entire population of women who appeared for their first prenatal appointment at the Mount Sinai Hospital OB/GYN Ambulatory Practice between February 3, 2020, and June 12, 2020. The Ambulatory Practice serves a population of primarily Hispanic and African-American minority women (90\%) who reside in the inner city. The remaining $10 \%$ of women self-identify as Asian, Caucasian, Filipino, Indian, Islander, Native American, and "unknown." Approximately 
Fig. 1 Observed EPDS average by week

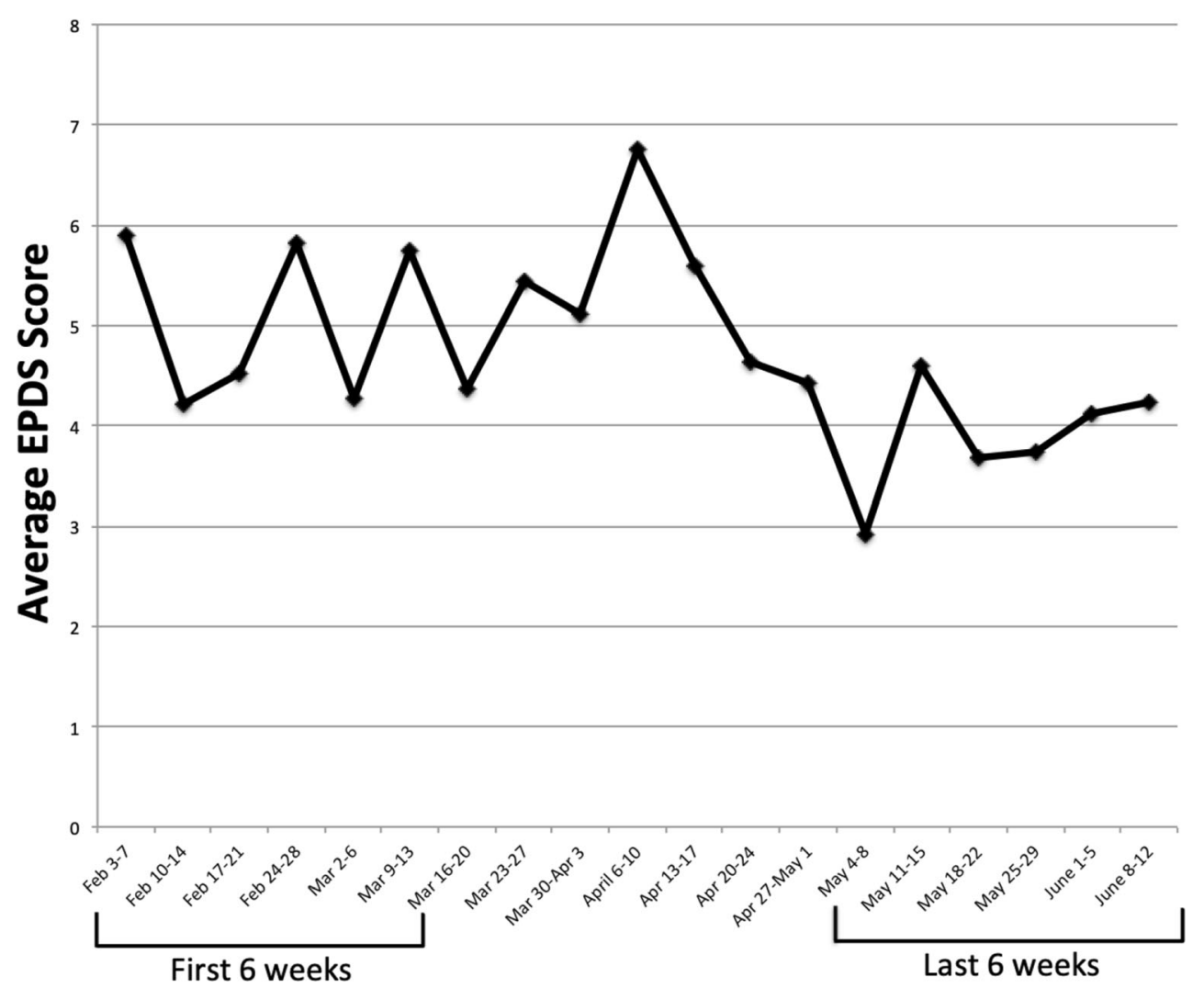

$70 \%$ of the women reported being single or unmarried at their first preterm appointment, and $90 \%$ were enrolled in a US government-funded healthcare plan.

\section{Mood assessment}

The Edinburgh Postnatal Depression Scale (EPDS; Cox et al. 1987) was provided to all women as a screen for prenatal mood dysregulation. The EPDS is a ten-item self-report multidimensional instrument, validated in over 36 languages, capable of assessing symptoms associated with depression and anxiety using a scale of 0-30. Initially designed to assess postpartum mood, the EPDS effectively assesses mood throughout pregnancy (Meijer et al. 2014). When used as a first step in screening for significant mood changes or when used in a retrospective study for statistical analysis, the recommended cutoff score of $\geq 9$ indicates symptomatology consistent with possible depression, whereas a cutoff score of $\geq$ 12 indicates probable depression, with a sensitivity of $86 \%$, and a specificity of 78\% (Cox et al. 1987). Whenever possible, assessments were provided in the patient's primary language.

\section{Analysis}

The patient's date of service and EPDS score was downloaded directly from the Mount Sinai Hospital network and imported into SAS 9.2 for analysis. To accomplish the study aims, two separate analyses were run. First, women were divided into groups of those screened before and those after New York's March 12, 2020, ban on large gatherings, and second, those who appeared and were screened during the 6 weeks before imposing social restrictions (February 2, 2020-March 11, 2020) and those appearing and screened during the last 6 weeks of available data (May 4, 2020-June 12, 2020).

All work was performed in compliance with the Mount Sinai School of Medicine Program for the Protection of Human Subjects and in accordance with the Health Insurance Portability and Accountability Act (HIPAA) security rule guidelines enacted in 2003 .

\section{Results}

Of the 488 women who appeared for their first prenatal appointment between February 3, 2020, and June 12, 2020, 485 women (ages 16-40 years) had recorded EPDS scores; three women declined EPDS screening. Of the women who completed the EPDS, $406(83.7 \%)$ reported some change of mood over the past 2 weeks (EPDS $\geq 1), 73$ women $(15.1 \%)$ had a score $\geq 9$, and 40 women $(8.2 \%)$ had a score $\geq 12$ (mean $=4.7$, median $=4$, range $=0-22, \mathrm{SD}=4.2$ ). While there was no significant difference observed in mean EPDS scores between women screened before $(n=153$; mean $=5.0$, median $=4$, range $=0-21, \mathrm{SD}=4.4)$ and following $(n=332 ;$ mean $=4.5$, 
Fig. 2 Observed prenatal appointments per week. No significant difference in prenatal appointments by week $(p=0.59$, n.s)

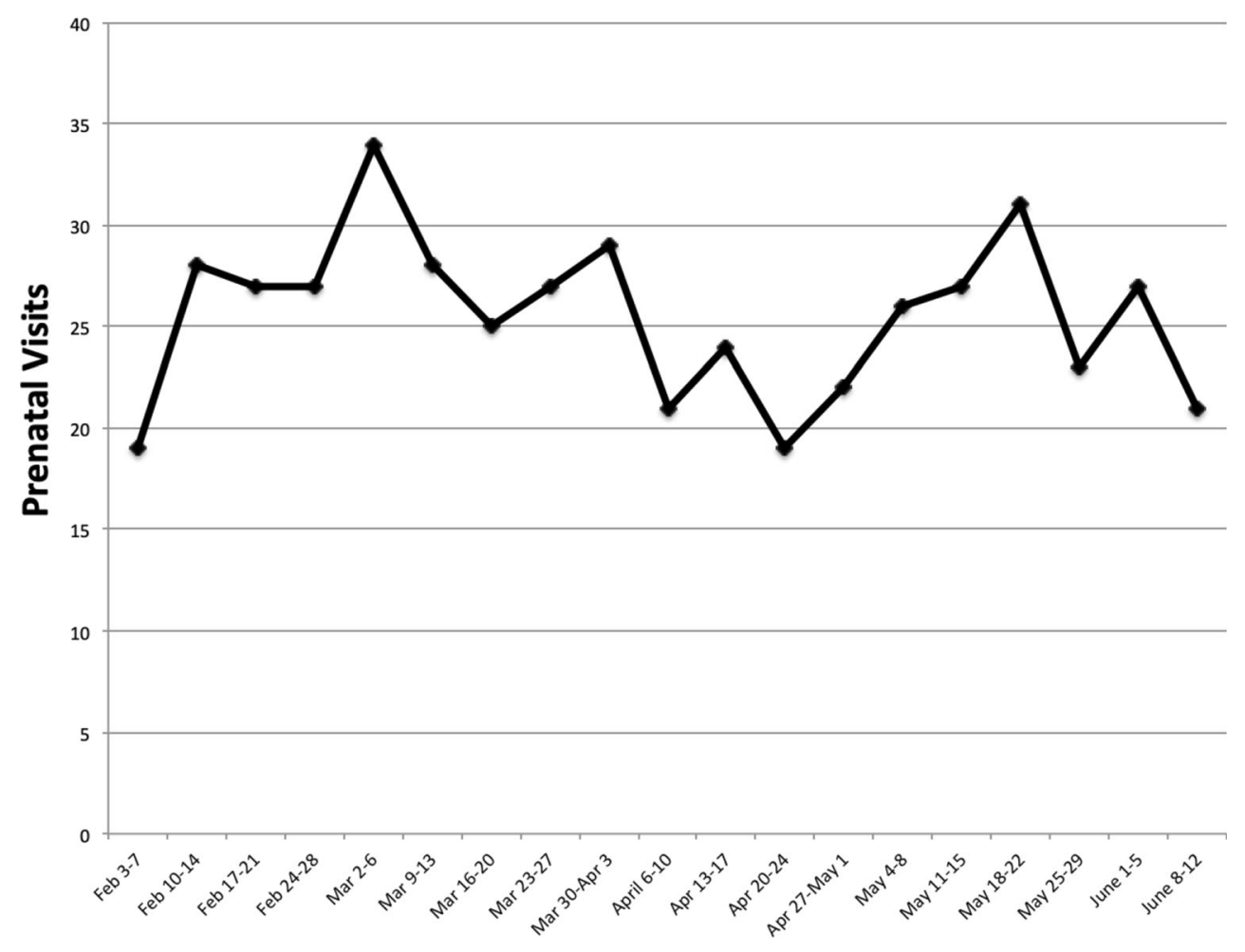

median $=4$, range $=0-22, \mathrm{SD}=4.0$ ) the March 12,2020 , restrictions, $t(483)=1.16, p=0.25$, n.s., a significant difference in EPDS scores were observed between those women who appeared for their first prenatal appointment in the 6 weeks prior to restrictions $(n=154$; mean $=5.0$, median $=4$, range $=$ $0-21, \mathrm{SD}=4.4)$ compared with those who appeared the last 6 weeks of available data, May 4, 2020-June 12, $2020(n=$ 155 ; mean $=3.9$, median $=3$, range $=0-14, \mathrm{SD}=3.6$ ), $t(307)=2.51, p<0.01$; corresponding to restrictions of schools and nonessential business. Notably, this difference is related to decreased symptomatology. (Fig. 1).

\section{Discussion}

Women living in low socioeconomic status in New York City demonstrated an improvement in mood in light of the implementation of social restrictions in New York City secondary to the COVID-19 pandemic. While the pandemic's effects on pregnant women are of considerable concern among clinicians who treat pregnant women, surprisingly, symptoms of mood dysregulation for minority women of low SES appeared to decline.

On March 12, 2020, the World Health Organization declared the outbreak of COVID-19 a global pandemic, and community-wide restrictions closing school and non-essential business were mandated across New York State. Although considerable efforts have focused on the medical challenges associated with COVID-19, less attention has been focused on the mental health implications of the "shut-down" during pregnancy. That we found lower rates of depression symptomatology among minority women is consistent with an increasing body of research demonstrating minority women living in low socioeconomic status may be less likely to suffer from acute episodes of depression (Bailey et al. 2019), may be more resilient to effects of stressful life events (Assari and Lankarani 2016), and may also be more emotionally resilient in the face of opportunistic illness (Dale and Safren 2018). Equally notable is that some consequences of imposed social restrictions may have had unexpected positive effects on health and wellbeing (see Nelson 2020), specifically among those living in lower SES. For example, the well-understood social and economic factors which disproportionately impact mothers living in low SES such as unavailable childcare, limited partner and family support, and reduced time flexibility secondary to formal and informal employment obligations, which undoubtedly play a role in contributing to poorer maternal mental health outcomes (Beeber et al. 2008), may have, in many cases, been ameliorated in light of the imposed social restrictions.

While these findings are important towards understanding the early maternal needs of those living in poverty, we recognize some critical limitations. First, the assessment used, the EPDS, a validated measure of mood change throughout pregnancy, may not be sensitive enough to detect symptoms associated specifically with COVID-19 pandemic concerns. However, the specificity and sensitivity to general symptoms of both anxiety (Matthey, 2008) and mood (Cox et al. 1987) as 
measured by the EPDS are high. Second, we did not analyze the data using demographic factors as covariates. Because there may be a subgroup of pregnant women disproportionally experiencing mood changes, this will need to be further explored as the days of the pandemic continue, and the sample size increases, thereby allowing for appropriate statistical power to determine such differences. Similarly, given the Mount Sinai Hospital OB/GYN Ambulatory Practice's prenatal EPDS screening program began shortly before the pandemic was announced, and as we presumably remain in the early stages of the crisis, it is also possible that as the pandemic continues and concerns associated with extended unemployment intensify, anxiety and depression among this population will increase. Finally, a problem universal to all health registry-based studies is that outcome variables may only represent treatment-seeking behavior, as opposed to incidence. That is, we can only assess those women who chose to come for their appointments, and given the increased concern of COVID-19 in medical settings, we realize the most anxious and concerned prenatal women may have chosen not to attend their appointments for fear of contracting the virus. Such an occurrence would result in an underestimation of symptoms and could explain why EPDS scores appeared to improve. To explore this possibility, we conducted a post hoc analysis of prenatal appointment attendance for each week to determine if there was a significant difference after restrictions were implemented. No difference was observed (Fig. 2).

Minority women living in low SES are among the most vulnerable for prenatal mood disruption. However, many of the social and economic disparities among those living in lower SES, which likely also play a contributing role to poorer maternal health outcomes, may have been reduced as a result of imposed social restrictions. That we found a decrease in symptomatology is also consistent with a recent Gallop Panel (2020) reporting that many Americans are experiencing less worry than they had earlier in the pandemic. While these findings have implications for urban health policy, as the pandemic continues, and as economic and employment concerns worsen, our results might prove temporary. Certainly, because we are still in the early stages of the pandemic and as maternal stress, anxiety, and depression may adversely affect the infant's long-term well-being (Kinsella and Monk 2009), monitoring pregnant women for emotional dysregulation continues to warrant special attention.

Authors' contributions All authors were involved in the study concept, design, and drafting of the manuscript. Ms. Burgos had full access to all of the data in the study. All authors take responsibility for the integrity of the data. Dr. Silverman was responsible for the accuracy of the data analysis.

Data availability N/A

\section{Compliance with ethical standards}

Conflicts of interest The authors declare that they have no conflict of interest.

Ethics approval This study was performed in compliance with the Mount Sinai School of Medicine Program for the Protection of Human Subjects and in accordance with the Health Insurance Portability and Accountability Act (HIPAA) security rule guidelines enacted in 2003.

Consent to participate N/A

Consent for publication N/A

Code availability N/A

\section{References}

Assari S, Lankarani MM (2016) Association between stressful life events and depression; intersection of race and gender. J Racial Ethn Health Disparities 3(2):349-356

Bailey RK, Mokonogho J, Kumar A (2019) Racial and ethnic differences in depression: current perspectives. Neuropsychiatr Dis Treat 15: 603-609

Beeber LS, Perreira KM, Schwartz T (2008) Supporting the mental health of mothers raising children in poverty: how do we target them for intervention studies? Ann N Y Acad Sci 1136:86-100

Cox JL, Holden JM, Sagovsky R (1987) Detection of postnatal depression. Development of the 10-item Edinburgh postnatal depression scale. Br J Psychiatry 150:782-786

Dale SK, Safren SA (2018) Resilience takes a village: black women utilize support from their community to foster resilience against multiple adversities. AIDS Care 30(sup5):S18-S26

Gallop Panel (2020) U.S. adults report less worry, more happiness. May 18, 2020. Retrieved from https://news.gallup.com/poll/311135/adultsreport-less-worry-happiness.aspx Accessed May 18, 2020

Kaiser Family Foundation poll (2020) The implications of COVID-19 for mental health and substance use. April 24, 2020 https://wwwkfforg/ coronavirus-covid-19/issue-brief/kff-health-tracking-poll-late-april2020/ Accessed May 16, 2020

Kinsella MT, Monk C (2009) Impact of maternal stress, depression and anxiety on fetal neurobehavioral development. Clin Obstet Gynecol 52(3):425-440

Loudon H, Nentin F, Silverman ME (2016) Using clinical decision support as a means of implementing a universal postpartum depression screening program. Arch Womens Ment Health 19(3):501-505

Matthey S (2008) Using the Edinburgh Postnatal Depression Scale to screen for anxiety disorders. Depress Anxiety. 25(11):926-931. https://doi.org/10.1002/da.20415

Meijer JL, Beijers C, van Pampus MG, Verbeek T, Stolk RP, Milgrom J, Bockting CL, Burger H (2014) Predictive accuracy of Edinburgh postnatal depression scale assessment during pregnancy for the risk of developing postpartum depressive symptoms: a prospective cohort study. BJOG 121(13):1604-1610

Nelson B (2020) The positive effects of covid-19. BMJ 369:m1785

Shakeel N, Eberhard-Gran M, Sletner L et al (2015) A prospective cohort study of depression in pregnancy, prevalence and risk factors in a multi-ethnic population. BMC Pregnancy Childbirth 15(1):5

Publisher's note Springer Nature remains neutral with regard to jurisdictional claims in published maps and institutional affiliations. 